\title{
Recent development in spinel cobaltites for supercapacitor application
}

\begin{abstract}
Precious metal oxides exhibit impressive characteristics that caught worldwide attention due to their promising capacitive performance, excellent electrochemical stability and low resistance, and these metal oxides have been extensively employed in supercapacitor application. This type of supercapacitors is known as redox supercapacitors or pseudocapacitors which applied faradaic process in storing energy in their systems. Thus, new materials with impressive electrochemical performance are highly demanded. In this aspect, cobaltite system with spinel structure has been the subject of intense research due to its established applications in electrochemistry. Besides, carbonaceous materials like activated carbons, carbon nanotubes, graphites, graphenes and fullerenes utilize electric double-layer capacitance whereby energy is stored by charge separation at an electrode/electrolyte interface. With greater development conducted on metal oxides and carbonaceous materials for supercapacitor application, introduction of hybrid and composite electrodes comprise of these two types of materials have been well received.
\end{abstract}

Keyword: B. Composites; D. Transition metal oxides; E. Electrodes; Supercapacitors 